\section{Unkomplizierte Harnwegsinfekte: Phytotherapie mit Mehrfacheffekt}

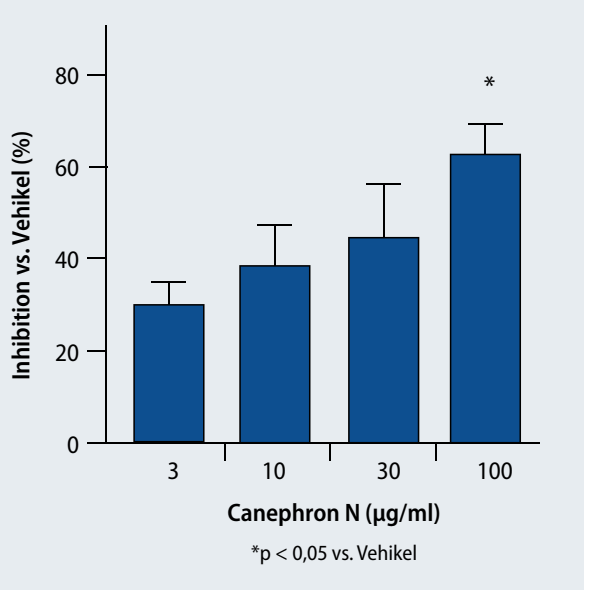

Bakterielle Adhäsion: Inhibition im Vergleich zum Vehikel (\%). Canephron ${ }^{\oplus} \mathrm{N}$ hindert Bakterien dosisabhängig daran, sich an das Urothel anzuheften.

— Die Phytomedizin ist eine wichtige Ressource für die Zukunft, berichtete Prof. André Gessner, Regensburg.

Sowohl in der Praxis als auch bei nosokomialen Infektionen führen ESKAPE-Bakterien zu großen Problemen. Unter dem Begriff werden sechs Bakterienarten zusammengefasst, die dem Selektionsdruck durch Antibiotika aufgrund von Multiresistenzen entkommen: Enterococcus faecium, Staphylococcus aureus, Klebsiella pneumoniae, Acinetobacter baumannii, Pseudomonas aeruginosa und Enterobakterium spp. Ein neues Antibiotikum mit befriedigendem Aktivitätsprofil sei derzeit nicht in Sicht. Als mögliche Alternative bieten pflanzliche Arzneimittel daher eine Chance, so Gessner.

Auch Prof. Kurt Naber, Straubing, Mitautor der aktuellen deutschen Leitlinien zur Therapie von Harnwegsinfekten, betonte, dass die antibiotische Therapie von unkomplizierten Harnwegsinfekten aufgrund der steigenden Resistenzsituation kritisch zu betrachten sei. Nicht zuletzt wegen der Resistenzproblematik und möglichen Nebenwirkungen der Antibiotikatherapie werden wirksame und verträgliche Alternativen wie zum Beispiel Phytotherapeutika untersucht. Die pflanzliche Kombination aus Liebstöckel, Tausendgüldenkraut und
Rosmarinblättern in Canephron ${ }^{\circledast} \mathrm{N}$ zeigte in einer Metaanalyse von 18 Studien mit über 3.100 Patienten eine gute Verträglichkeit und Sicherheit, berichtete Naber. Der antiphlogistische Effekt von Canephron ${ }^{\circledR} \mathrm{N}$ sorgt für eine Linderung von Brennen und Entzündungsschmerz. Die spasmolytische Wirkung entspannt die Blase, das Druckgefühl und die Krämpfe lassen nach. Ferner hilft die diuretische Wirkung, die Keime auszuspülen. Die antibakteriellen Effekte hemmen die Bakterienvermehrung.

Kürzlich wurde in vitro zudem eine anti-adhäsive Wirkung gezeigt, die die Bakterien daran hindert, sich an das Urothel anzuheften [Künstle et al. EAU-Kongress Mailand 2013, Poster 671]. Die Wirkung war dosisabhängig und erreichte bei der höchsten Dosis eine Hemmwirkung von $61 \%$ gegenüber dem Vehikel (s. Abbildung). All diese Effekte führten zu einer im Akutfall rasch symptomlindernden und über längere Zeit Rezidive verhindernden Wirkung. Friederike Klein

Wissenschaftliches Symposium „80 years of Bionorica - Phytoneering one Step ahead in thinking and research!" Berlin, 27. Februar 2013. Veranstalter: Bionorica, Neumarkt

\title{
Ejaculatio praecox: Therapiemöglichkeiten noch zu wenig bekannt
}

— Eine Ejaculatio praecox (EP) hat eine Prävalenz von circa $20 \%$ und ist damit bei Männern häufiger als ein Diabetes mellitus, erklärte Prof. Hartmut Porst, Hamburg. Definitionsgemäß bedeutet eine EP: Ejakulation innerhalb von weniger als zwei Minuten nach Penetration, keine Kontrolle über den Samenerguss und hoher Leidensdruck. Unterschieden wird zwischen einer lebenslangen (primären) EP mit genetischem Hintergrund, von der rund zwei Drittel der Patienten betroffen sind und die bereits ab Beginn der sexuellen Aktivitäten besteht, und einer erworbenen (sekundären) EP, die sich in der Regel erst nach vielen Jahren entwickelt, häufig in Verbindung mit Erektionsstörungen oder anderen Erkrankungen wie Prostatitis oder Hyperthyreose. An der Entstehung der primären EP sind mit großer Wahrscheinlichkeit Fehlfunktionen der Serotonin-Rezeptoren und Störungen der Serotoninhomöostase beteiligt, berichtete Porst.

In der Praxis wird die EP selten thematisiert. Die meisten Betroffenen suchen erst nach langer Leidensdauer Hilfe und treffen dann oft auf Ärzte, denen das Krankheitsbild kaum vertraut ist, so die Erfahrung von Porst. Abhilfe schaffen soll ein neuer Leitfaden, in dem die verschiedenen EP-Formen, das Diagnosekonzept und die Therapieoptionen erörtert werden (ab Mai 2013 über den Außendienst von Berlin-Chemie erhältlich). Einziges bei EP zugelassenes Medika- ment ist Dapoxetin (Priligy ${ }^{\circledast}$ ), ein kurz wirksamer selektiver Serotonin-Wiederaufnahme-Hemmer (SSRI) zur Bedarfsmedikation. Die Wirksamkeit des Medikaments wurde in fünf klinischen Phase-III-Studien mit insgesamt über 6.000 Patienten belegt. Die intravaginale Ejakulationslatenzzeit (IELP) wurde fast vervierfacht, von rund einer $\mathrm{Mi}$ nute zu Beginn bis auf knapp vier Minuten unter $60 \mathrm{mg}$ Dapoxetin. Mit zunehmender Behandlungsdauer wurden die Therapieeffekte stärker, berichtete Dr. Thomas Kreutzig, Freiburg, der wie Porst an dem Expertenkonsens beteiligt war. Deshalb sollte man bei einem Therapieversuch "die Flinte nicht zu früh ins Korn werfen“. Roland Fath

Symposium „Ejaculatio praecox: 1, 2, 3 ... schon vorbei!“ im Rahmen des 119. Kongresses der Deutschen Gesellschaft für Innere Medizin (DGIM), Wiesbaden, 7. April 2013.

Veranstalter: Berlin-Chemie, Berlin 